\title{
Mirage mediation from the landscape
}

\author{
Howard Baer $\odot,{ }^{1, *}$ Vernon Barger, ${ }^{2, \dagger}$ and Dibyashree Sengupta $\odot^{1, \hbar}$ \\ ${ }^{1}$ Homer L. Dodge Department of Physics and Astronomy, University of Oklahoma, Norman, Oklahoma 73019, USA \\ ${ }^{2}$ Department of Physics, University of Wisconsin, Madison, Wisconsin 53706, USA
}

(Received 17 December 2019; accepted 4 February 2020; published 19 March 2020)

\begin{abstract}
Rather general considerations from the string theory landscape suggest a statistical preference within the multiverse for soft supersymmetry (SUSY) breaking terms as large as possible subject to a pocket universe value for the weak scale not greater than a factor of 2-5 from our measured value. Within the gravity/mod.u.lusmediated SUSY breaking framework, the Higgs mass is pulled to $m_{h} \simeq 125 \mathrm{GeV}$ while first/second generation scalars are pulled to tens of $\mathrm{TeV}$ scale and gauginos and third generation scalars remain at the few $\mathrm{TeV}$ range. In this case, one then expects comparable modulus- and anomaly-mediated contributions to soft terms, leading to mirage mediation. For an assumed stringy natural value of the SUSY $\mu$ parameter, we evaluate predicted sparticle mass spectra for mirage mediation from a statistical scan of the string landscape. We then expect a compressed spectrum of gauginos along with a higgsino-like lightest-SUSY particle. For a linear (quadratic) statistical draw with gravitino mass $m_{3 / 2} \sim 20 \mathrm{TeV}$, then the most probable mirage scale is predicted to be around $\mu_{\text {mir }} \sim 10^{13}$ $\left(10^{14}\right) \mathrm{GeV}$. SUSY should appear at high-luminosity large hadron collider via higgsino pair production into soft dilepton pairs. Distinguishing mirage mediation from models with unified gaugino masses may have to await construction of an international linear collider with $\sqrt{s}>2 m$ (higgsino).
\end{abstract}

DOI: 10.1103/PhysRevResearch.2.013346

\section{INTRODUCTION}

So far, our only plausible understanding for the tiny, yet nonzero, value of the cosmological constant $\Lambda$ comes from Weinberg's multiverse explanation [1,2]. Assuming a vast array of pocket universes (PUs) within a broader multiverse [3], each with different physical laws, then it may not be surprising that we observe $\Lambda \sim 10^{-120} m_{P}^{4}$ (where $m_{P}$ is the reduced Planck mass $m_{P}=2.4 \times 10^{18} \mathrm{GeV}$ ) since if its value was much larger, then the cosmic expansion would be so fast that galaxies could not condense, and observers would likely not arise. This anthropic explanation depends on assuming a fertile patch of pocket universes within the multiverse which all have the Standard model (SM) as the low-energy effective theory but for which the values of the cosmological constant are spread uniformly across the decades of possible values [4]. Such reasoning allowed Weinberg to predict the value of $\Lambda$ to be within a factor of a few, many years before its value was actually measured. These arguments were bolstered by the emergence of flux compactifications within string theory which provided the needed discretuum of metastable pocket universes within the broader multiverse [5-7].

It is reasonable to ask if other scales, such as the weak scale, might arise from anthropic reasoning [8,9]. In this case,

\footnotetext{
*baer@ou.edu

†barger@pheno.wisc.edu

†ibyashree.Sengupta-1@ou.edu
}

Published by the American Physical Society under the terms of the Creative Commons Attribution 4.0 International license. Further distribution of this work must maintain attribution to the author(s) and the published article's title, journal citation, and DOI. it is usually assumed that the weak scale effective theory of the fertile patch is the softly broken minimal supersymmetric standard model (MSSM) so that the weak scale is protected from large quantum mechanical corrections. In the context of string theory, typically a variety of hidden sectors appear and several $F$ - and $D$-term supersymmetry (SUSY) breaking vacuum expectation values (vevs) can contribute to the overall SUSY breaking scale [10] which then in turn determines the weak scale via the scalar potential minimization conditions. With a possibly nonminimal hidden sector, and with SUSY breaking vevs uniformly distributed across the decades of possible values, then statistically large overall SUSY breaking scales are favored. Naively, one might expect as well that large values of the weak scale would also be favored. Douglas has suggested a power-law statistical distribution for the overall soft SUSY breaking scale $m_{\text {soft }}$ of the form $m_{\text {soft }}^{n}$, where $n=$ $2 n_{F}+n_{D}-1, n_{F}$ is the number of $F$ - breaking fields, and $n_{D}$ is the number of $D$-breaking fields contributing to the overall SUSY breaking scale [10].

Several factors intervene to counteract this expectation [11]. First, electroweak symmetry must be properly broken: no charge or color breaking vacua (CCB) are allowed. Second, the soft terms must be such that $m_{H_{u}}^{2}$ is driven radiatively to negative values so that EW symmetry is actually broken. Third, the pocket universe value of the weak scale $m_{\text {weak }}^{\mathrm{PU}}$ should be within a factor of a few from our measured value of the weak scale. Nuclear physics computations from Agrawal et al. [8] have shown that if $m_{\text {weak }}^{\mathrm{PU}} \gtrsim(2-5) m_{\text {weak }}^{\text {OU }}$ (OU stands for our universe) then stable nucleons are all $\Delta^{++}$baryons. Complex nuclei will not form and consequently atoms as we know them will not form in such a universe. This anthropic requirement is known as the atomic principle in that in order to have a universe with observers, then likely atoms (and 
consequently chemistry) as we understand them would have to be formed [12].

This picture has been explored in the context of gravitymediated SUSY breaking models in the three-extra-parameter nonuniversal Higgs model (NUHM3) [13]. Motivated by the fact that all chiral matter superfields live in a 16-plet of $\mathrm{SO}(10)$, the parameters of the NUHM3 model [14] include a common scalar mass for the first two generations $m_{0}(1,2)$ with a separate mass for the third generation $m_{0}(3)$. The Higgs superfields obtain independent soft terms $m_{H_{u}}^{2}$ and $m_{H_{d}}^{2}$ while the gauginos are unified at $m_{1 / 2}$ at the GUT scale. There are also common trilinear soft terms $A_{0}$ and bilinear $B$ although $B$ is usually traded for the ratio of Higgs vevs $\tan \beta \equiv v_{u} / v_{d}$ via the scalar potential minimization conditions. It is also more convenient to trade the GUT scale values of $m_{H_{u}}^{2}$ and $m_{H_{d}}^{2}$ for the weak scale values of the (SUSY conserving) Higgs/higgsino $\mu$ parameter and the weak scale value of the pseudoscalar Higgs mass $m_{A}$. The superparticle mass spectrum of the gravity-mediated NUHM3 model can then be calculated from the final form of the NUHM3 model parameter space:

$$
m_{0}(1,2), m_{0}(3), m_{1 / 2}, A_{0}, \tan \beta, \mu, m_{A} \quad \text { (NUHM3). }
$$

The approach of calculating SUSY particle mass spectra from the combined statistical draw to large soft terms along with an anthropic veto of vacua with improper EWSB or else too large a value of $m_{\text {weak }}^{\mathrm{PU}}$ has met with some considerable success. In Ref. [11], a qualitative examination was made and the flow of soft terms towards preferred statistical/anthropic values was shown to favor a large value of light Higgs mass $m_{h} \sim 125 \mathrm{GeV}$ while at the same time radiatively driving the weak scale soft terms towards natural values comparable to the measured weak scale. In Ref. [13], a more quantitative approach generated probability distributions for Higgs and sparticle masses for the mild $n=1$ and 2 statistical draw to large soft terms. The Higgs mass probability histogram was found to peak at $m_{h} \simeq 125 \mathrm{GeV}$ which was understood in part because $A_{0}$ is pulled as large as possible but stopping short of CCB minima- this lifts $m_{h}$ to $\sim 125 \mathrm{GeV}$ due to large mixing in the stop sector and hence large radiative corrections to $m_{h}$. Sparticle masses were typically pulled beyond large hadron collider (LHC) reach. In Ref. [15], the SUSY landscape spectra from NUHM3 was confronted by various LHC sparticle and Higgs search limits and by WIMP dark matter search limits. Typical spectra would lie beyond both accelerator and dark matter search limits. In Ref. [16], in the context of SUSY axion models, it was shown that the draw to large $\mathrm{PQ}$ sector soft terms pulled one also to a large value of the PQ scale $f_{a}$. A large value of $f_{a}$ would generate too much axion dark matter and too much WIMP dark matter due to late time axino and saxion decays in the early universe. It was concluded that the PQ sector soft terms were likely correlated with MSSM soft terms so that dark matter would not be overproduced. In Ref. [17], it was shown that the draw to large first/second generation scalars could solve the SUSY flavor problem via a mixed decoupling/degeneracy solution since both first and second generation scalars would be drawn to large but common upper bounds in the $20-30 \mathrm{TeV}$ region.
In this paper, we extend this methodology to mixed gravity/moduli plus anomaly-mediated soft SUSY breaking (SSB) terms [18] in the context of the natural generalized mirage mediation model (nGMM) [19]. Since the draw to large soft terms is related to a draw to large gravitino masses in supergravity, then we would expect a gravitino mass $m_{3 / 2}$ in the tens of $\mathrm{TeV}$ regime from SUSY on the landscape. However, gaugino, third generation, and Higgs soft terms contribute to the weak scale either directly or via 1-loop terms and so must instead lie in the $\mathrm{TeV}$, not tens of $\mathrm{TeV}$, regime. In such circumstances, then one would expect comparable anomaly-mediated and modulus-mediated (MM) contributions to soft terms - a situation which requires mirage mediated rather then gravity-mediated only values for soft terms [18].

Our plan for this paper is then as follows. In the next Sec. IA, we present a very brief review of some previous work on mirage-mediated SUSY breaking. Then, in Sec. II, we present the nGMM soft terms and parameter space and explain our methodology for drawing the modulus-mediated soft terms to large values compared to the compulsory AMSB soft terms [20] which only depend on the gravitino mass. In Sec. III, we present histograms of probability for the various nGMM parameters. These results include a prediction of the mirage scale $\mu_{\text {mir }}$ where gaugino masses are expected to unify. The mirage scale prediction depends on the assumed value of $m_{3 / 2}$ and on the assumed power $n$ of the power-law selection of soft SUSY breaking terms from the landscape. We also present probability histograms for the various sparticle and Higgs masses. By measuring the gaugino masses directly at colliders such as LHC or indirectly via splittings amongst the light higgsino masses at a linear $e^{+} e^{-}$collider [21], then the mirage scale can be determined by running the weak scale gaugino masses up to the mirage scale [22]. Once the moduli/AMSB mixing parameter $\alpha$ is determined, then the associated gravitino mass $m_{3 / 2}$ can be determined. This in turn allows one to match against the predicted histograms for the mirage scale for a particular value of $m_{3 / 2}$. We present a summary and conclusions in Sec. IV.

\section{A. Brief review of some previous work on mirage mediation}

The original mirage mediation scheme grew out of the Kachru-Kallosh-Linde-Trivedi (KKLT) proposal [23] for modulus stabilization accompanied by some uplifting mechanism to gain a de Sitter minimum, i.e., a small cosmological constant from the landscape. The KKLT proposal was made in the context of IIB string theory compactified on an orientifold containing D3 and D7 branes. The complex structure or shape moduli and the dilaton could be stabilized by introducing NS and RR three-form fluxes with masses near the string scale. A remaining single Kähler modulus $T$ would be stabilized by nonperturbative effects such as gaugino condensation or brane instantons, with $m_{T} \sim m_{3 / 2} \ln \left(m_{P} / m_{3 / 2}\right)$, leading to a supersymmetric AdS vacuum. As a final step, an uplifting mechanism-here the addition of an anti-D3 brane near the tip of a Klebanov-Strassler throat-would raise the scalar potential of the theory to gain a de Sitter vacuum with softly broken $N=1$ supersymmetry. 
In the KKLT scheme, a little hierarchy

$$
m_{T} \sim\left(4 \pi^{2}\right) m_{3 / 2} \sim\left(4 \pi^{2}\right) m_{\mathrm{soft}}
$$

was expected to ensue [18,24], where $\ln \left(m_{P} / m_{3 / 2}\right) \sim 4 \pi^{2}$ and $m_{\text {soft }}$ is the expected scale of modulus (gravity) mediated soft terms. Since $m_{\text {soft }}$ was suppressed relative to $m_{3 / 2}$, then the modulus-mediated soft terms are expected to be comparable to contributions from anomaly mediation [which are suppressed relative to $m_{3 / 2}$ by $\sim 1 /\left(16 \pi^{2}\right)$ loop factor]. The resultant model has been dubbed mirage mediation [25] (MM) due to the distinctive feature that gaugino (and scalar) masses evolve from nonuniversal values at the GUT scale to apparently universal values at some intermediate scale

$$
\mu_{\text {mir }}=m_{\mathrm{GUT}} e^{\left(-8 \pi^{2} / \alpha\right)}
$$

where the introduced parameter $\alpha$ measures the relative modulus-mediated versus anomaly-mediated contributions to gaugino masses [26,27].

Upon integrating out the heavy dilaton field and the shape moduli, one is left with an effective broken supergravity theory of the observable sector fields denoted by $\hat{Q}$ and the size modulus field $\hat{T}$. The Kähler potential depends on the location of matter and Higgs superfields in the extra dimensions via their modular weights $n_{i}=0$ (1) for matter fields located on $D 7$ (D3) branes, or $n_{i}=1 / 2$ for chiral multiplets on brane intersections, while the gauge kinetic function $f_{a}=$ $\hat{T}^{l_{a}}$, where $a$ labels the gauge group, is determined by the corresponding location of the gauge supermultiplets, since the power $l_{a}=1$ (0) for gauge fields on $D 7$ (D3) branes [26,27].

Within the MM model, the SSB gaugino mass parameters, trilinear SSB parameters and sfermion mass parameters, all renormalized just below the unification scale (taken to be $Q=$ $\left.m_{\mathrm{GUT}}\right)$, are given by

$$
\begin{gathered}
M_{a}=M_{s}\left(l_{a} \alpha+b_{a} g_{a}^{2}\right), \\
A_{i j k}=M_{s}\left(-a_{i j k} \alpha+\gamma_{i}+\gamma_{j}+\gamma_{k}\right), \\
m_{i}^{2}=M_{s}^{2}\left(c_{i} \alpha^{2}+4 \alpha \xi_{i}-\dot{\gamma}_{i}\right),
\end{gathered}
$$

where $M_{s} \equiv \frac{m_{3 / 2}}{16 \pi^{2}}, b_{a}$ are the gauge $\beta$ function coefficients for gauge group $a$, and $g_{a}$ are the corresponding gauge couplings. The coefficients that appear in (4)-(6) are given by $c_{i}=1-n_{i}, a_{i j k}=3-n_{i}-n_{j}-n_{k}$, and $\xi_{i}=\sum_{j, k} a_{i j k} \frac{y_{i j k}^{2}}{4}-$ $\sum_{a} l_{a} g_{a}^{2} C_{2}^{a}\left(f_{i}\right)$. Finally, $y_{i j k}$ are the superpotential Yukawa couplings, $C_{2}^{a}$ is the quadratic Casimir for the ath gauge group corresponding to the representation to which the sfermion $\tilde{f}_{i}$ belongs, $\gamma_{i}$ is the anomalous dimension, and $\dot{\gamma}_{i}=8 \pi^{2} \frac{\partial \gamma_{i}}{\partial \ln \mu}$. Expressions for the last two quantities involving the anomalous dimensions can be found in Appendices of Refs. [27,28].

The MM model is then specified by the parameters

$$
m_{3 / 2}, \alpha, \tan \beta, \operatorname{sgn}(\mu), n_{i}, l_{a} .
$$

The mass scale for the SSB parameters is dictated by the gravitino mass $m_{3 / 2}$. The phenomenological parameter $\alpha$, which could be of either sign, determines the relative contributions of anomaly mediation and gravity mediation to the soft terms, and is expected to be $|\alpha| \sim \mathcal{O}(1)$. Grand unification implies matter particles within the same GUT multiplet have common modular weights, and that the $l_{a}$ are universal. We will assume here that all $l_{a}=1$ and, for simplicity, there is a common modular weight for all matter scalars $c_{m}$ but we will allow for different modular weights $c_{H_{u}}$ and $c_{H_{d}}$ for each of the two Higgs doublets of the MSSM. Such choices for the scalar field modular weights are motivated for instance by SO(10) SUSY GUT models where the MSSM Higgs doublets may live in different ten-dimensional Higgs reps.

Various aspects of MM phenomenology have been examined in Refs. [26,27,29-31]. Of recent importance is to confront the MM models for various modular weight choices with the LHC Higgs mass discovery and also sparticle mass constraints. By scanning over MM models with different $n_{m}$ and $n_{H}$ modular weight choices, but requiring $m_{h}=125 \pm$ $2 \mathrm{GeV}$, then all models were found to be rather highly finetuned in the electroweak using the conservative $\Delta_{\mathrm{EW}}$ measure of fine-tuning [32].

The electroweak fine-tuning parameter $[33,34], \Delta_{\mathrm{EW}}$, is a measure of the degree of cancellation between various contributions on the right-hand side (RHS) in the well-known expression for the $Z$ mass:

$$
\begin{aligned}
\frac{m_{Z}^{2}}{2} & =\frac{m_{H_{d}}^{2}+\Sigma_{d}^{d}-\left(m_{H_{u}}^{2}+\Sigma_{u}^{u}\right) \tan ^{2} \beta}{\tan ^{2} \beta-1}-\mu^{2} \\
& \simeq-m_{H_{u}}^{2}-\Sigma_{u}^{u}-\mu^{2}
\end{aligned}
$$

which results from the minimization of the Higgs potential in the MSSM. Here, $\tan \beta=v_{u} / v_{d}$ is the ratio of Higgs field vacuum-expectation values and the $\Sigma_{u}^{u}$ and $\Sigma_{d}^{d}$ contain an assortment of radiative corrections, the largest of which typically arise from the top squarks. Expressions for the $\Sigma_{u}^{u}$ and $\Sigma_{d}^{d}$ are given in Appendix of Ref. [34]. If the RHS terms in Eq. (8) are individually comparable to $m_{Z}^{2} / 2$, then no unnatural fine-tunings are required to generate $m_{Z}=91.2 \mathrm{GeV} . \Delta_{\mathrm{EW}}$ is defined to be the largest of these terms, scaled by $m_{Z}^{2} / 2$. Clearly, low electroweak fine-tuning requires that $\mu$ be close to $m_{Z}$ and that $m_{H_{u}}^{2}$ be radiatively driven to small negative values close to the weak scale. This scenario has been dubbed radiatively driven natural supersymmetry or RNS [33,34].

While the various MM models with particular discrete modular weight choices seem inconsistent with LHC Higgs mass measurements and sparticle mass limits, many general features of mirage mediation models were found to occur in a variety of different string based models. First, while the SSB terms were calculated within KKLT inspired setups including a single Kähler modulus $T$, realistic string compactifications typically contain $\mathcal{O}(10-100)$ Kähler moduli. Under more general (and more plausible) compactifications, then it is reasonable to expect the general MM pattern of soft terms to ensue, but where the discrete modular weight choices are replaced by continuous parameters. For this reason, in Ref. [19] a generalized mirage mediation model (GMM) was proposed with continuous rather than discrete parameter choices, as detailed in Sec. II. The continuous parameters could allow for large trilinear soft terms $A_{0}$ which are needed to lift $m_{h}$ to $\sim 125 \mathrm{GeV}$ while reducing fine-tuning in the $\Sigma_{u}^{u}\left(\tilde{t}_{1,2}\right)$ terms. Also, the increased flexibility of GMM allowed for small $\mu \sim 100-300 \mathrm{GeV}$ as expected from naturalness.

In addition, a wide variety of models was found to contain features of MM, but with important differences in the scalar sector. Indeed, Choi and Nilles [35] emphasize that 
the MM pattern of gaugino masses is rather general in a wide class of string-motivated models whilst deviations in the scalar sector are to be expected. Examples include heterotic compactifications with a partially sequestered uplifting sector [36], IIB theory with large volume compactifications [37,38] (where gauginos are expected to adopt the mirage pattern but scalar masses are expected $\sim m_{3 / 2}$ ) and heterotic orbifold compactifications [39-42]. These latter models exhibit the phenomena of local grand unification [43] wherein different orbifold locations exhibit different gauge symmetries. For instance, first and second generation matter superfields may lie at orbifold fixed points and so occur in complete 16dimensional SO(10) representations with SSB scalar masses of order $m_{3 / 2}$. In contrast, the gauginos, Higgs superfields and third generation matter live more in the bulk, thus occuring in split representations and with SSB masses of order $m_{3 / 2} /\left(4 \pi^{2}\right)$ and where the gaugino masses are expected with the mirage form. Thus the sparticle mass spectra is expected to reflect the geography of fields on the particular compactification manifold; this scheme is expected to be a more general result than just that which arises from any particular orbifold which has been selected [44]. The expected collider and dark matter phenomenology of such models has been exhibited in Ref. [45]. In addition, expectations for SUSY from 11D $M$-theory models compactified on a manifold of $G_{2}$ holonomy predict scalar masses of order $m_{3 / 2} \sim 50-100 \mathrm{TeV}$ but with suppressed gaugino masses [46]. In such a case, one also expects comparable anomaly- and modulus-mediated contributions to soft SUSY breaking terms.

\section{METHODOLOGY}

In our approach, we will adopt the form of soft SUSY breaking terms expected from general mirage mediation [19] with a parameter space given by

$$
\alpha, m_{3 / 2}, c_{m}, c_{m 3}, a_{3}, c_{H_{u}}, c_{H_{d}}, \tan \beta \quad(\mathrm{GMM}),
$$

where $a_{3}$ is short for $a_{Q_{3} H_{u} U_{3}}$ [appearing in Eq. (5)] and $c_{m}, c_{m 3}$, $c_{H_{u}}$, and $c_{H_{d}}$ arise in Eq. (6). Here, we adopt an independent value $c_{m}$ for the first two matter-scalar generations whilst the parameter $c_{m 3}$ applies to third generation matter scalars. In the GMM model, the $a_{i j k}$ and $c_{i}$ are elevated from discrete to continuous parameters in order to accommodate more general string theories and more general compactification schemes. The independent values of $c_{H_{u}}$ and $c_{H_{d}}$, which set the modulusmediated contribution to the soft Higgs mass-squared soft terms, may conveniently be traded for weak scale values of $\mu$ and $m_{A}$ as is done in the two-parameter nonuniversal Higgs model (NUHM2) [14]:

$$
\alpha, m_{3 / 2}, c_{m}, c_{m 3}, a_{3}, \tan \beta, \mu, m_{A}\left(\mathrm{GMM}^{\prime}\right) .
$$

This procedure allows for more direct exploration of stringy natural SUSY parameter space where most landscape solutions require $\mu \sim 100-300 \mathrm{GeV}$ in anthropically allowed pocket universes [47].

Thus our final formulas for the soft terms are given by

$$
\begin{gathered}
M_{a}=\left(\alpha+b_{a} g_{a}^{2}\right) m_{3 / 2} / 16 \pi^{2}, \\
A_{\tau}=\left(-a_{3} \alpha+\gamma_{L_{3}}+\gamma_{H_{d}}+\gamma_{E_{3}}\right) m_{3 / 2} / 16 \pi^{2},
\end{gathered}
$$

$$
\begin{aligned}
& A_{b}=\left(-a_{3} \alpha+\gamma_{Q_{3}}+\gamma_{H_{d}}+\gamma_{D_{3}}\right) m_{3 / 2} / 16 \pi^{2}, \\
& A_{t}=\left(-a_{3} \alpha+\gamma_{Q_{3}}+\gamma_{H_{u}}+\gamma_{U_{3}}\right) m_{3 / 2} / 16 \pi^{2}, \\
& m_{i}^{2}(1,2)=\left(c_{m} \alpha^{2}+4 \alpha \xi_{i}-\dot{\gamma}_{i}\right)\left(m_{3 / 2} / 16 \pi^{2}\right)^{2}, \\
& m_{j}^{2}(3)=\left(c_{m 3} \alpha^{2}+4 \alpha \xi_{j}-\dot{\gamma}_{j}\right)\left(m_{3 / 2} / 16 \pi^{2}\right)^{2}, \\
& m_{H_{u}}^{2}=\left(c_{H_{u}} \alpha^{2}+4 \alpha \xi_{H_{u}}-\dot{\gamma}_{H_{u}}\right)\left(m_{3 / 2} / 16 \pi^{2}\right)^{2}, \\
& m_{H_{d}}^{2}=\left(c_{H_{d}} \alpha^{2}+4 \alpha \xi_{H_{d}}-\dot{\gamma}_{H_{d}}\right)\left(m_{3 / 2} / 16 \pi^{2}\right)^{2},
\end{aligned}
$$

where for a given value of $\alpha$ and $m_{3 / 2}$, the values of $c_{H_{u}}$ and $c_{H_{d}}$ are adjusted so as to fulfill the input values of $\mu$ and $m_{A}$. In the above expressions, the index $i$ runs over first/second generation MSSM scalars $i=$ $Q_{1,2}, U_{1,2}, D_{1,2}, L_{1,2}$, and $E_{1,2}$ while $j$ runs overs third generation scalars $j=Q_{3}, U_{3}, D_{3}, L_{3}$, and $E_{3}$. The natural GMM model has been incorporated into the event generator program ISAJET 7.86 [48] which we use here for spectra generation.

Douglas has proposed that the distribution of multiverse vacua versus hidden sector mass scale $m_{\text {hidden }}$ with a given value of the weak scale $m_{\text {weak }}$ is represented by [10]

$$
\begin{aligned}
& d N_{\text {vac }}\left[m_{\text {hidden }}^{2}, m_{\text {weak }}, \Lambda\right] \\
& \quad=f_{\text {SUSY }}\left(m_{\text {hidden }}^{2}\right) \cdot f_{\text {EWSB }} \cdot f_{\mathrm{CC}} \cdot d m_{\text {hidden }}^{2},
\end{aligned}
$$

where the soft SUSY breaking terms are related to the hidden sector mass scale as $m_{\text {soft }} \sim m_{\text {hidden }}^{2} / m_{P} \sim m_{3 / 2}$ and CC stands for the Cosmological Constant. With many hidden sectors possible in string theory, then $m_{\text {hidden }}^{4}=\Sigma_{i}\left|F_{i}\right|^{2}+\sum_{\alpha} D_{\alpha}^{2}$ for the various $F$ and $D$ terms contributing to the totality of the SUSY breaking scale. Douglas observed that with $\hat{\Lambda}=$ $3 e^{K}|W|^{2}$ being the norm of the superpotential, then the cosmological constant is

$$
\Lambda=\sum_{i}\left|F_{i}\right|^{2}+\sum_{\alpha} D_{\alpha}^{2}-\hat{\Lambda} .
$$

Since the superpotential $W$ receives additive contributions from many sectors of the theory, both supersymmetric and nonsupersymmetric - then one expects a uniform distribution in $\hat{\Lambda}$ and hence scanning in this variable fixes the cosmological constant to an anthropic value independent of the SUSY breaking scale. Thus we may expect $f_{\mathrm{CC}} \sim \Lambda / m_{\text {string }}^{4}$.

In addition, the total SUSY breaking scale is given by the distance from the origin in the space of all SUSY breaking parameters-and in a high-dimensional space, most of the volume is near the boundary. With a uniform distribution of individual SUSY breaking parameters, then one expects a power law draw towards large SUSY breaking scale:

$$
f_{\text {SUSY }} \sim\left(m_{\text {Soft }}\right)^{2 n_{F}+n_{D}-1},
$$

where $n_{F}$ is the number of $F$-term breaking fields and $n_{D}$ is the number of $D$-term breaking fields, and the factor of 2 arises because the $F$-breaking fields are complex whilst the $D$-breaking fields are real. Using this rather general ansatz, then already SUSY breaking by a single $F$ term implies a linear statistical draw to large soft terms. For multiple SUSY breaking fields, then the draw to large soft terms is even stronger. 
An initial suggestion [49] for $f_{\mathrm{EWSB}}$ was that $f_{\mathrm{EWSB}} \sim$ $\left(m_{\text {weak }} / m_{\text {soft }}\right)^{2}$ which would reflect the overall trend of multiplying the distribution by a naturalness measure. While this ansatz favors soft terms not too far removed from the weak scale, it fails in a number of cases [13]. For instance, if trilinear soft terms become too big, one is forced into chargeand/or-color breaking minima of the Higgs potential. Such vacua must be vetoed rather than penalized by a statistical factor. Also, if $m_{H_{u}}^{2}$ becomes too large, then EW symmetry does not even break-again, such vacua must be vetoed.

Here, we will follow the nuclear physics results of Agrawal et al. [8] who found that in various pocket universes within our fertile patch of MSSM effective theories, the generated value of the weak scale must be within a factor 2-5 of our measured value, lest all nucleons turn into $\Delta^{++}$baryons in which case complex nuclei, and hence atoms, will no longer form. Thus, here we obey the atomic principle [12]: that complex life as we know it requires the existence of atoms, and consequently chemistry.

To accommodate different weak scale values $m_{\text {weak }}^{\text {PU }}$ in different pocket universes, we invert the usual usage of Eq. (8). We assume a natural solution to the SUSY $\mu$ problem (such as the hybrid CCK or hybrid SPM models presented in Ref. [50] which generate a gravity-safe $\mathrm{U}(1)_{P Q}$ symmetry for solving the strong CP problem at the same time as generating $R$-parity conservation) ${ }^{1}$ with $\mu \sim 200 \mathrm{GeV}$. (If $\mu \gg m_{\text {weak }}$, then only a relatively tiny fraction of vacua lead to $m_{\text {weak }} \sim 100 \mathrm{GeV}$ [47].) Then, instead of fixing $m_{Z}$ at its measured value in our universe, we can calculate its pocket universe value $m_{Z}^{\mathrm{PU}}$ for a given set of soft terms. We will require a value of $m_{Z}^{\mathrm{PU}}<4 m_{Z}$ (our universe) in accord with Agrawal et al. which then corresponds to a value of $\Delta_{\mathrm{EW}}<30$. In this case, even with appropriate EWSB, large $A_{0}$ and $m_{H_{u}}^{2}$ terms actually lead to smaller contributions to the weak scale rather than larger ones (until one is forced into CCB or no EWSB vacua: see Refs. $[11,47])$. Thus, for $f_{\text {EWSB }}$, we will adopt

$$
f_{\text {EWSB }}=\Theta\left(30-\Delta_{\text {EW }}\right)
$$

where each scan point leads to a different value of $m_{Z}^{\mathrm{PU}}$.

To begin our scan over $\mathrm{GMM}^{\prime}$ parameter points, we proceed as follows. (1) We select a particular value of $m_{3 / 2}$ which then fixes the AMSB contributions to SSB terms. (2) We also fix $\mu=200 \mathrm{GeV}$ for a natural solution to the SUSY $\mu$ problem. This then allows for arbitrary values of $m_{Z}^{\mathrm{PU}}$ to be generated but disallows any possibility of fine-tuning $\mu$ to gain $m_{Z}^{\mathrm{OU}}$.

Next, we will invoke Douglas' power-law selection of modulus-mediated soft terms relative to AMSB contributions within the GMM model. Thus, for an assumed value of $n=$ $2 n_{F}+n_{D}-1$, we will generate the following. (1) $\alpha^{n}$ with $\alpha$ : $3-25$, a power-law statistical selection for modulus-mediated gaugino masses $M_{a},(a=1-3)$ over the gauge groups; (2) $\left(a_{3} \alpha\right)^{n}$, a power-law statistical selection of modulus-mediated $A$ terms, with $\left(a_{3} \alpha\right): 3-75$; (3) $m_{0}(1,2) \sim m_{3 / 2}$ so that $c_{m}=$ $\left(16 \pi^{2} / \alpha\right)^{2}$ so that first/second generation scalars are set

${ }^{1}$ For a review of twenty solutions to the SUSY $\mu$ problem, see Ref. [51]. maximally at $m_{3 / 2} ;(4)\left(\sqrt{c_{m 3} \alpha^{2}}\right)^{n}$ to gain a power-law statistical selection on third generation scalar masses $m_{0}(3)$, with $\left(\sqrt{c_{m 3} \alpha^{2}}\right): 3-80 ;(5)$ a power-law statistical selection on $m_{H_{d}}^{2}$ via $m_{A}^{n}$ with $m_{A}: 300-7000 \mathrm{GeV}$; and (6) a uniform selection on $\tan \beta: 3-40$.

Our first informative scan allows us to narrow the range of $\alpha$ and $\sqrt{c_{m 3} \alpha^{2}}$ while expanding the range of $a_{3} \alpha, m_{A}$, and $\tan \beta$. Our second scan proceeds with (1) $\alpha^{n}$ with $\alpha$ : 5-20, a power-law statistical selection for modulus-mediated gaugino masses $M_{a},(a=1-3)$ over the gauge groups; (2) $\left(a_{3} \alpha\right)^{n}$, a power-law statistical selection of modulus-mediated $A$ terms, with $\left(a_{3} \alpha\right): 3-100$; (3) $m_{0}(1,2) \sim m_{3 / 2}$ so that $c_{m}=\left(16 \pi^{2} / \alpha\right)^{2}$ so that first/second generation scalars are set maximally at $m_{3 / 2}$; (4) $\left(\sqrt{c_{m 3} \alpha^{2}}\right)^{n}$ to gain a power-law statistical selection on third generation scalar masses $m_{0}(3)$, with $\left(\sqrt{c_{m 3} \alpha^{2}}\right)$ : 30-60; (5) a power-law statistical selection on $m_{H_{d}}^{2}$ via $m_{A}^{n}$ with $m_{A}: 300-10000 \mathrm{GeV}$; and (6) a uniform selection on $\tan \beta: 3-50$, followed by a focused scan by generating (1) $\alpha^{n}$ with $\alpha$ : 5-20, a power-law statistical selection for modulus-mediated gaugino masses $M_{a},(a=1-3)$ over the gauge groups; (2) $\left(a_{3} \alpha\right)^{n}$, a power-law statistical selection of modulus-mediated $A$ terms, with $\left(a_{3} \alpha\right): 3-75$; (3) $m_{0}(1,2) \sim m_{3 / 2}$ so that $c_{m}=\left(16 \pi^{2} / \alpha\right)^{2}$ so that first/second generation scalars are set maximally at $m_{3 / 2}$; (4) $\left(\sqrt{c_{m 3} \alpha^{2}}\right)^{n}$ to gain a power-law statistical selection on third generation scalar masses $m_{0}(3)$, with $\left(\sqrt{c_{m 3} \alpha^{2}}\right)$ : 30-60; (5) a power-law statistical selection on $m_{H_{d}}^{2}$ via $m_{A}^{n}$ with $m_{A}: 1000-7000 \mathrm{GeV}$; and (6) a uniform selection on $\tan \beta: 3-40$.

We adopt a uniform selection on $\tan \beta$ since this parameter is not a soft term. Note that with this procedure-while arbitrarily large soft terms are statistically favored-in fact they are all bounded from above since once they get too big, they will lead either to nonstandard EW vacua or else too large a value of $m_{Z}^{\mathrm{PU}}$. In this way, models such as split SUSY or high scale SUSY would be ruled out since for a natural value of $\mu$, then they would necessarily lead to $m_{Z}^{\mathrm{PU}} \gg(2-5) m_{Z}^{\mathrm{OU}}$.

\section{RESULTS FOR MIRAGE MEDIATION FROM THE MULTIVERSE}

In the following figures, we scan the soft terms of the $\mathrm{GMM}^{\prime}$ model according to the power law $m_{\text {soft }}^{n}$ for $n=1$ and 2 with a fixed gravitino mass $m_{3 / 2}=20 \mathrm{TeV}$. Proceeding with much higher values of $m_{3 / 2} \gtrsim 25 \mathrm{TeV}$ always results in too-large of contributions to the weak scale when we take $m_{0}(1,2) \simeq m_{3 / 2}$ (see Fig. 10 of Ref. [45]). We keep $\mu$ fixed at $200 \mathrm{GeV}$ according to a natural solution to the SUSY $\mu$ problem. We also veto nonstandard EW vacua while for vacua with appropriate EWSB we require $f_{\mathrm{EWSB}}=\Theta\left(30-\Delta_{\mathrm{EW}}\right)$ which corresponds to $m_{Z}^{\mathrm{PU}} \leqslant 4 m_{Z}^{\mathrm{OU}}$. This latter anthropic selection imposes an upper bound on most GMM' parameters and sparticle masses which would otherwise increase without limit according to $f_{\text {SUSY }}$.

\section{A. Parameters}

In Fig. 1, we first show the normalized probability histogram $d P / d \alpha$ as a function of $\alpha$. The histogram is normalized to unit area. We also show for convenience on the upper 


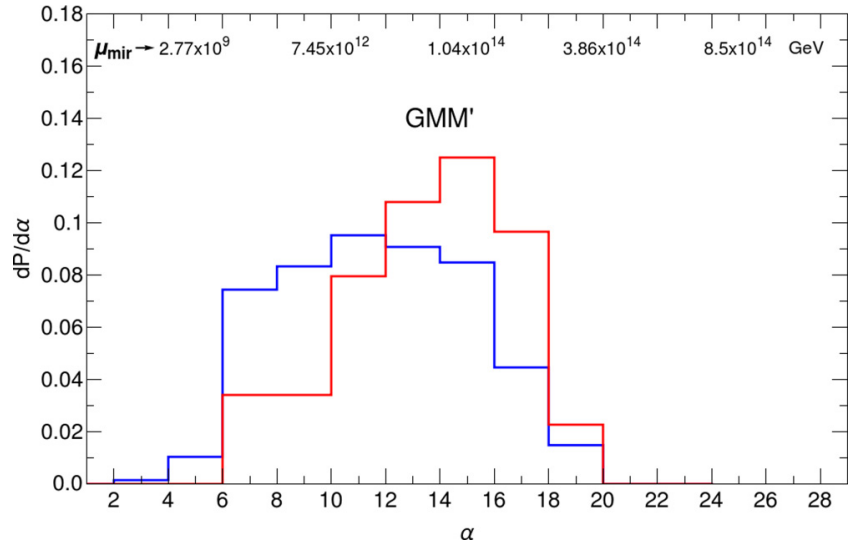

FIG. 1. Probability distribution for mixed modulus-anomaly mixing parameter $\alpha$ from $n=1$ (blue) and $n=2$ (red) statistical scans over the $\mathrm{GMM}^{\prime}$ model with $m_{3 / 2}=20 \mathrm{TeV}$.

scale various corresponding values of the gaugino mirage unification scale $\mu_{\mathrm{mir}}$. From the figure, for a simple linear draw ( $n=1$ corresponding to SUSY breaking from a single $F$ term), we see that the blue histogram has a rather broad peak spanning between $\alpha \sim 6-16$ which then corresponds to a predicted mirage scale $\mu_{\text {mir }} \sim 10^{10}-10^{14} \mathrm{GeV}$. There is relatively little probability for $\mu_{\text {mir }} \lesssim 10^{9} \mathrm{Gev}$ or for $\mu_{\text {mir }} \gtrsim$ $2 \times 10^{14} \mathrm{GeV}$. The mirage scale is actually testable in the GMM model since if we measure any two of the three gaugino masses at the weak scale, then using the known RGEs [52], we can extrapolate up in energy to see where they intersect. An intersection of all three gaugino masses at some intermediate mass scale would be strong supporting evidence for mirage mediation and would pick off the requisite value of $\alpha$.
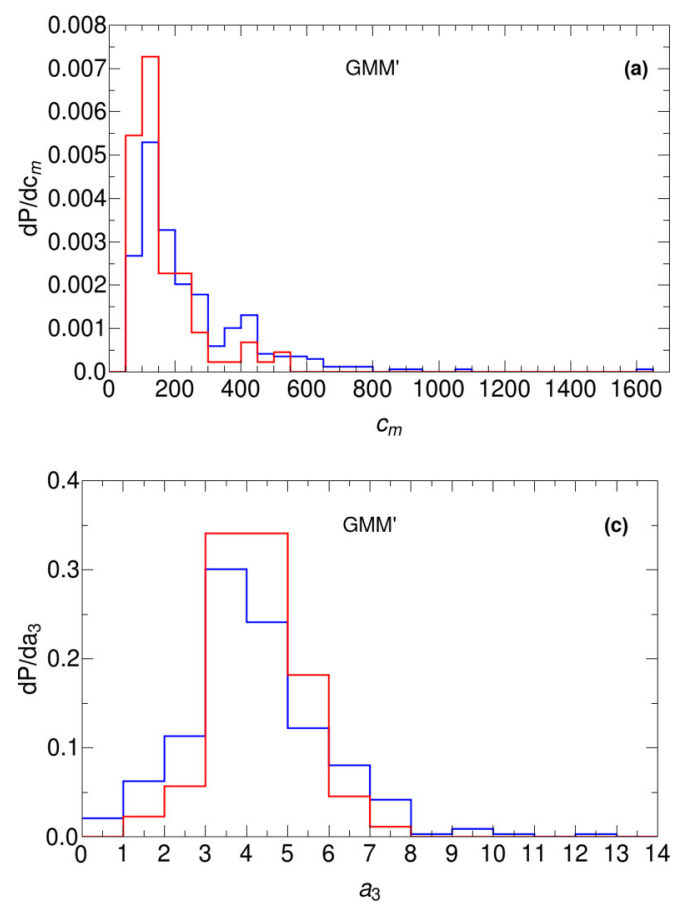

If instead we hypothesize an $n=2$ draw on soft terms, then we arrive at the red histogram. Here we see that the stronger statistical draw on modulus-mediated soft terms results in a preference for higher $\alpha$ values peaked now at $\alpha \sim 15$ corresponding to $\mu_{\text {mir }} \sim 10^{14} \mathrm{GeV}$. Substantial probability remains for $\mu_{\text {mir }}$ as low as $10^{11} \mathrm{GeV}$.

In Fig. 2, we show histograms of probability for the other remaining parameters. In frame (a), we show $d P / d c_{m}$ which peaks for values of $c_{m} \sim 100-150$ for both $n=1$ and $n=2$. Since we have required $c_{m}=\left(16 \pi^{2} / \alpha\right)^{2}$, this distribution just reflects the inverse-square distribution of $\alpha$ already shown in Fig. 1. In frame (b), we show the distribution in $c_{m 3}$. In this case, we find values of $c_{m 3}$ peaking at $c_{m 3} \sim 5-15$ which sets the third generation matter scalar masses. These are more tightly restricted by the landscape since they largely determine the $\Sigma_{u}^{u}\left(\tilde{t}_{1,2}\right)$ contributions to the weak scale. Since we cannot tune these away, then if they are too large we would have $m_{Z}^{\mathrm{PU}} \gtrsim$ $4 m_{Z}^{\mathrm{OU}}$ and we would violate the nuclear physics results of Ref. [8].

In frame (c), we show the distribution in $a_{3}$ which sets the magnitude of the modulus-mediated contribution to the trilinear soft term $A_{0}$. Here, we find a statistical draw to large $-A_{0}$ terms with $a_{3}$ peaking around 3-6. Such large $A_{t}$ terms actually reduce the weak scale contributions $\Sigma_{u}^{u}\left(\tilde{t}_{1,2}\right)[33,47]$. At the same time, large $A_{t}$ terms yield maximal mixing in the stop sector leading to an uplift of $m_{h}$ to $\sim 125 \mathrm{GeV}[53,54]$. If the $a_{3}$ parameter gets too big, then again large $\Sigma_{u}^{u}\left(\tilde{t}_{1,2}\right)$ terms result while if even large values of $a_{3}$ occur then we are pushed into CCB vacua (which must be vetoed).

In frame (d), we plot the distribution in $\tan \beta$, which was scanned uniformly. Here, we see the most probable value is $\tan \beta \sim 8-20$. For larger values of $\tan \beta \sim 20-50$, then the
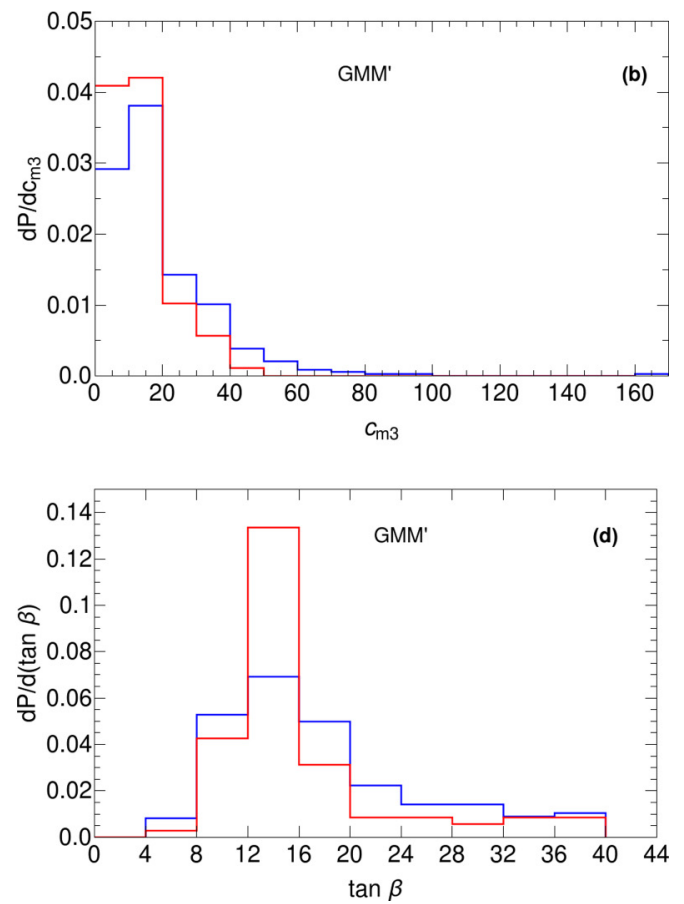

FIG. 2. Distributions in (a) $c_{m}$, (b) $c_{m 3}$, (c) $a_{3}$, and (d) $\tan \beta$. Here, $n=1$ (blue) and $n=2$ (red) are from statistical scans over the nGMM model with $m_{3 / 2}=20 \mathrm{TeV}$. 


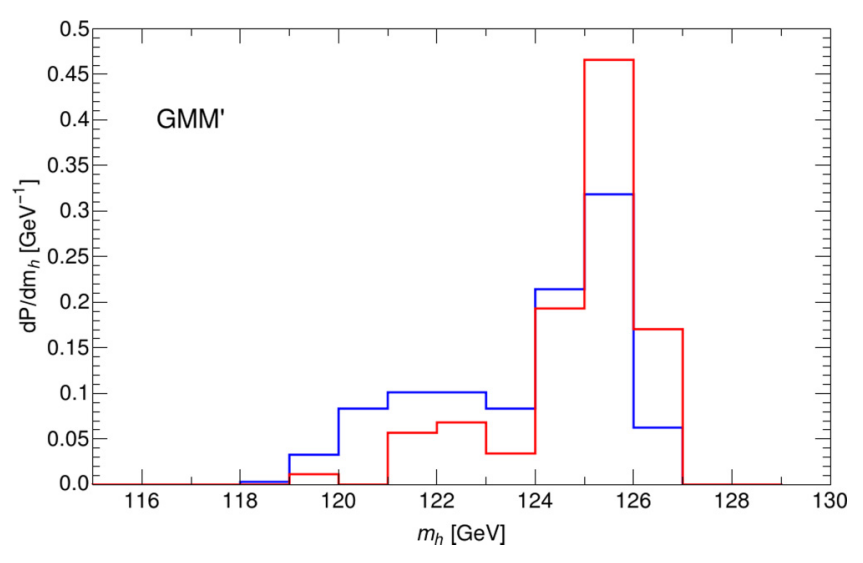

FIG. 3. Probability distribution for mass of light Higgs boson $m_{h}$ from $n=1$ (blue) and $n=2$ (red) statistical scans over nGMM model with $m_{3 / 2}=20 \mathrm{TeV}$.

$\tau$ and $b$-Yukawa couplings become large leading to large $\Sigma_{u}^{u}\left(\tilde{b}_{1,2}\right)$ contributions to the weak scale.

\section{B. Higgs and sparticle mass predictions}

In Fig. 3, we show the Higgs mass $m_{h}$ probability distribution from the GMM model in the landscape for $m_{3 / 2}=20 \mathrm{TeV}$ with $n=1$ (blue) and $n=2$ (red). From the plot, we see that the most probable value of $m_{h}$ is $125 \mathrm{GeV}$ for both cases. The value of $m_{h}$ reaches maximally $127 \mathrm{GeV}$ but much higher values of $m_{h}$ always require $m_{Z}^{\mathrm{PU}}>4 m_{Z}^{\mathrm{OU}}$ from the $\Sigma_{u}^{u}\left(\tilde{t}_{1,2}\right)$ contributions to the weak scale. These distributions are highly encouraging post-dictions of the Higgs mass from general considerations of the string landscape!

In Fig. 4(a), we show the probability distribution for $m_{\tilde{g}}$ from the landscape within generalized mirage mediation.
Here, we see that for $n=1$ with $m_{3 / 2}=20 \mathrm{TeV}$, then $m_{\tilde{g}} \sim$ 2-5 TeV, almost always safely beyond LHC Run 2 limits. For the $n=2$ case, then the distribution in $m_{t g}$ becomes somewhat harder with $m_{\tilde{g}} \sim 2.5-5 \mathrm{TeV}$ with a most-probable value of $m_{\tilde{g}} \sim 4 \mathrm{TeV}$. From these distributions, it seems reasonable that LHC has not yet discovered SUSY via gluino pair production. The HL-LHC reach extends to $m_{\tilde{g}} \sim 2.7 \mathrm{TeV}$ [55] while HE-LHC with $\sqrt{s}=27 \mathrm{TeV}$ will have a reach in $m_{\tilde{g}}$ to about $6 \mathrm{TeV}$ [56]. Thus discovery of SUSY via gluino pair production may have to await a higher energy upgrade of LHC [57].

In Fig. 4(b), we show the probability distribution for $m_{\tilde{t}_{1}}$. Here, we see for both $n=1$ and $n=2$ statistical draw, then $m_{\tilde{t}_{1}} \sim 1-2 \mathrm{TeV}$. These values of $m_{\tilde{t}_{1}}$ are generally beyond current LHC top-squark mass limits and so again it may be no surprise that LHC has not yet seen a signal via top-squark pair production. While HL-LHC should have a reach in $m_{\tilde{t}_{1}}$ to about $1.5 \mathrm{TeV}$, the reach of HE-LHC extends to about $m_{\tilde{t}_{1}} \sim 3 \mathrm{TeV}$ [56]. Thus it may well require an energy upgrade of LHC to discover SUSY via top-squark pair production.

In Fig. 4(c), we show the distribution in $m_{\tilde{t}_{2}}$. In this case, we expect the landscape with GMM to yield a value $m_{\tilde{t}_{2}} \sim$ 2.5-5 TeV. Typically, we expect the higher range of these values to be beyond the reach of even HE-LHC.

In Fig. 4(d), we show the expected probability for the pseudoscalar Higgs mass $m_{A}$. We find that $m_{A} \sim 2-6 \mathrm{TeV}$. Such values are typically beyond the reach of HL-LHC [58].

One of the features of mirage mediation is the expected compressed spectra of gauginos as compared to models with unified gaugino masses. For unified gauginos, we expect weak scale gaugino masses in the ratio $M_{1}: M_{2}: M_{3} \sim 1: 2: 6-7$. For the GMM model, these ratios can be quite different. The SU(3) gaugino mass $M_{3} \sim m_{\tilde{g}}$ (up to loop corrections) so that the approximate value of $M_{3}$ is given in Fig. 4(a). In Fig. 5, we
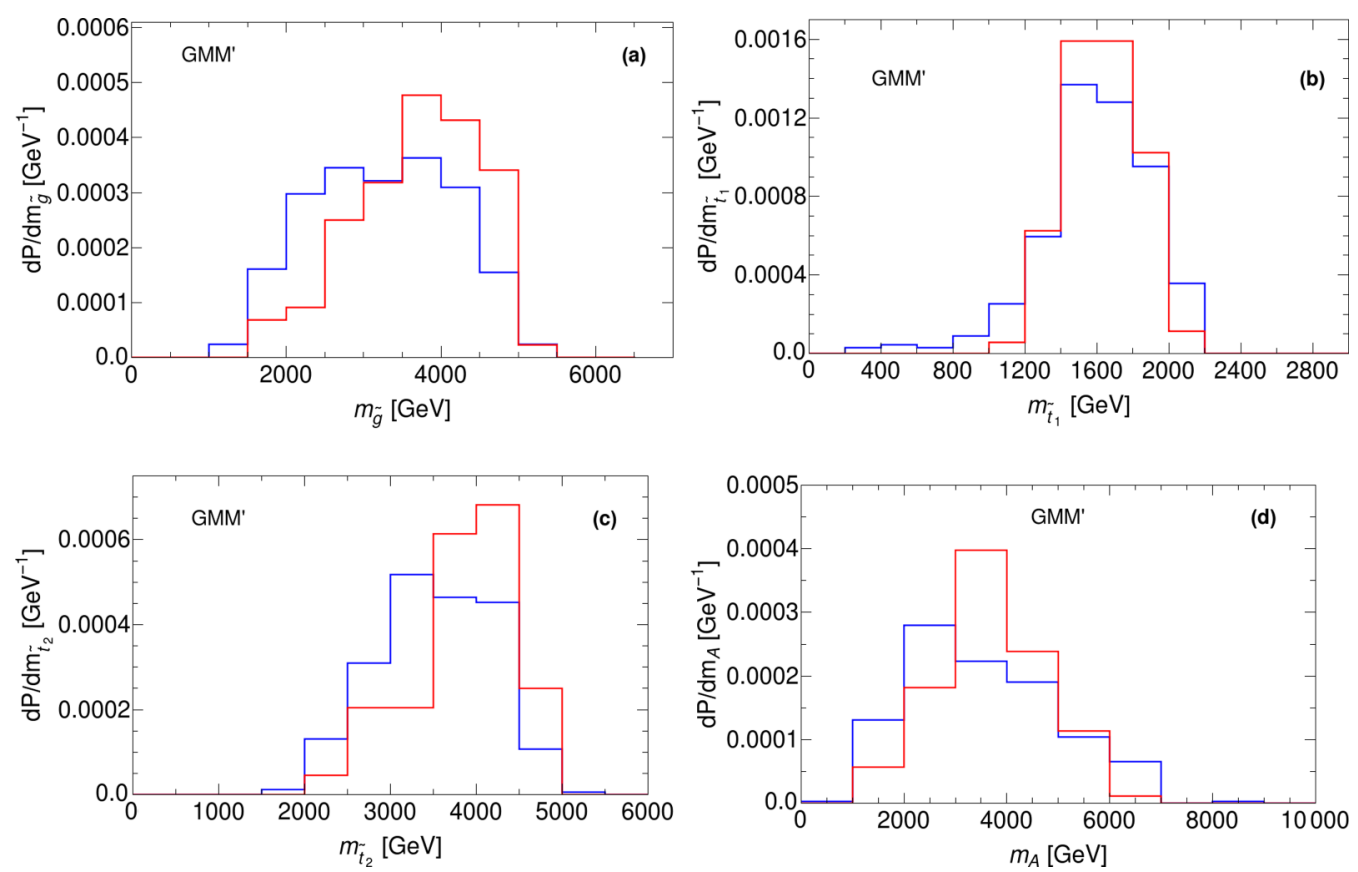

FIG. 4. Distributions in (a) $m_{\tilde{g}}$, (b) $m_{\tilde{t}_{1}}$, (c) $m_{\tilde{t}_{2}}$, and (d) $m_{A}$. Here, $n=1$ (blue) and $n=2$ (red) are from a statistical scans over the nGMM model with $m_{3 / 2}=20 \mathrm{TeV}$. 

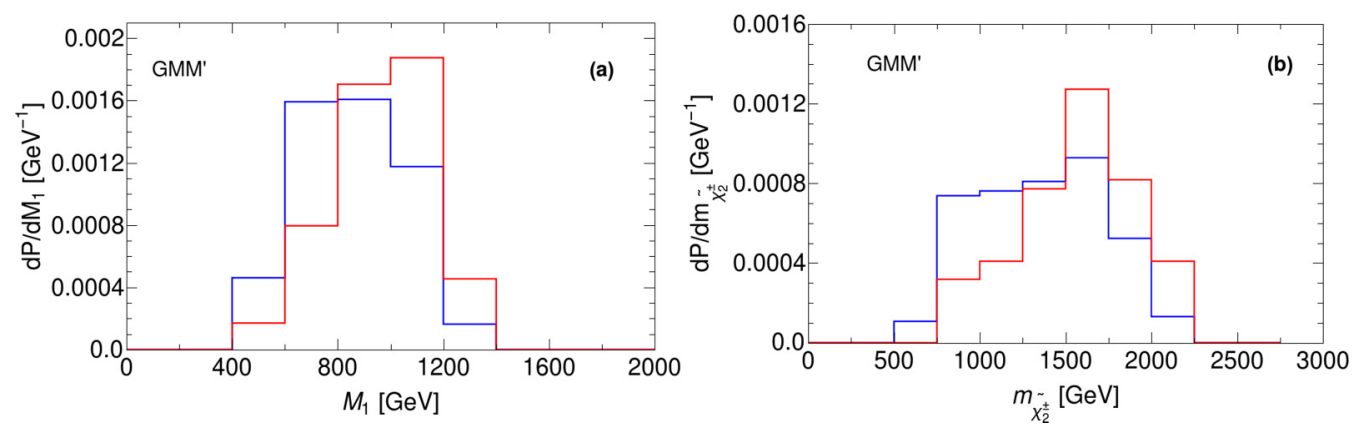

FIG. 5. Distributions in (a) $M_{1}$ and (b) $M_{2} \sim m_{\tilde{\chi}_{2}^{ \pm}}$. Here, $n=1$ (blue) and $n=2$ (red) are from statistical scans over the nGMM model with $m_{3 / 2}=20 \mathrm{TeV}$.

show the expected electroweak gaugino masses. In frame (a), the predicted bino mass $M_{1} \sim 0.5-1.3 \mathrm{TeV}$. This value is well above the expected value of $\mu \sim 100-350 \mathrm{GeV}$ and so we would expect the lightest-SUSY-particle (LSP) to be higgsinolike. The bino will be difficult to extract at LHC. However, a linear $e^{+} e^{-}$collider with $\sqrt{s}>2 m$ (higgsino) should be able to pair produce higgsinos via reactions such as $e^{+} e^{-} \rightarrow \tilde{\chi}_{1}^{0} \tilde{\chi}_{2}^{0}$ and measure the mass splitting $m_{\tilde{\chi}_{2}^{0}}-m_{\tilde{\chi}_{1}^{0}}$ which is sensitive to the bino mass [59]. Such a machine should be able to extract $M_{1}$ to test the distribution in Fig. 5(a). In Fig. 5(b), we show the wino mass $M_{2}$ probability distribution. It is expected that $M_{2} \sim 0.8-2.2 \mathrm{TeV}$. The LHC can access wino pair production $\tilde{\chi}_{2}^{ \pm} \tilde{\chi}_{4}^{0}$ via the same-sign diboson signature [60,61] (SSdB) which is unique to SUSY models with light higgsinos: $p p \rightarrow \tilde{\chi}_{2}^{ \pm} \tilde{\chi}_{4}^{0} \rightarrow W^{ \pm} W^{ \pm}+E_{T}$. The clean signature and signal production rate may allow one to extract a measurement of $M_{2}$ at HL- or HE-LHC via the total SSdB production rate. Otherwise, again an $e^{+} e^{-}$collider should be able to extract $M_{2}$ via the higgsino mass splittings which are measureable in higgsino pair production reactions [59].

In Fig. 6, we show the expected weak scale gaugino mass ratios (a) $M_{2} / M_{1}$ and (b) $M_{3} / M_{1}$ which are expected from the landscape with mirage mediation. From frame (a), we see that $M_{2} / M_{1}$ is expected to occur with ratio $\sim 1.4-1.7$ so that indeed the electroweakinos are compressed, but not highly compressed. Such a compressed gaugino mass spectrum would be solid evidence for mirage mediation [35]. In frame (b), we find that $M_{3} / M_{1} \sim 3-4$ rather than the expectation from gauginounified models where $M_{3} / M_{1} \sim 6-7$. While the gaugino mass spectrum is compressed, the gap $m_{\tilde{g}}-m_{\mathrm{LSP}}$ is actually greater than in gaugino-unified models since the LSP is higgsinolike and close to the weak scale whilst gluinos are pulled statistically to large values.

We also plot in Fig. 7 the expected $m_{\tilde{\chi}_{2}^{0}}-m_{\tilde{\chi}_{1}^{0}}$ mass gap. This gap is expected to be directly measurable at LHC via the higgsino pair production reaction $p p \rightarrow \tilde{\chi}_{1}^{0} \tilde{\chi}_{2}^{0}$ followed by $\tilde{\chi}_{2}^{0} \rightarrow \tilde{\chi}_{1}^{0} \ell^{+} \ell^{-}$[62]. (Indeed, there appears already some excess in this channel at Atlas with $139 \mathrm{fb}^{-1}$; see Fig. 10(a) of Ref. [63].) From the plot, we see the mass gap is typically $m_{\tilde{\chi}_{2}^{0}}-m_{\tilde{\chi}_{1}^{0}} \sim 4-12 \mathrm{GeV}$ so the opposite-sign (OS) dileptons will likely be quite soft. This discovery channel for SUSY appears to be the most propitious one for HL-LHC [15].

\section{C. $m_{0}^{\mathrm{MM}}$ versus $m_{1 / 2}^{\mathrm{MM}}$ parameter space for $m_{3 / 2}=20 \mathrm{TeV}$}

A panoramic view of some of our essential conclusions may be displayed in the $m_{0}^{\mathrm{MM}}$ versus $m_{1 / 2}^{\mathrm{MM}}$ plane which is then analogous to the $m_{0}$ versus $m_{1 / 2}$ plane of the mSUGRA/CMSSM or NUHM2,3 models. Here, we define $m_{0}^{\mathrm{MM}}=\sqrt{c_{m}} \alpha\left(m_{3 / 2} / 16 \pi^{2}\right)$ which is the pure modulusmediated contribution to scalar masses. The modulusmediated contribution to gaugino masses is correspondingly given by $m_{1 / 2}^{\mathrm{MM}} \equiv \alpha m_{3 / 2} /\left(16 \pi^{2}\right)$.

In Fig. 8(a), we show the $m_{0}^{\mathrm{MM}}$ versus $m_{1 / 2}^{\mathrm{MM}}$ plane for an $n=1$ landscape draw but with $a_{3}=1.6 \sqrt{c_{m}}$, with $c_{m}=c_{m 3}$ and with $\tan \beta=10, m_{A}=2 \mathrm{TeV}$, and $\mu=200 \mathrm{GeV}$. The lower-left yellow region shows where $m_{\widetilde{\chi}_{1}^{ \pm}}<103.5 \mathrm{GeV}$ in violation of LEP2 constraints. Also, the lower-left orange box shows where $\Delta_{\mathrm{BG}}<30$ (old naturalness calculation). The bulk of the low $m_{1 / 2}$ region here leads to tachyonic
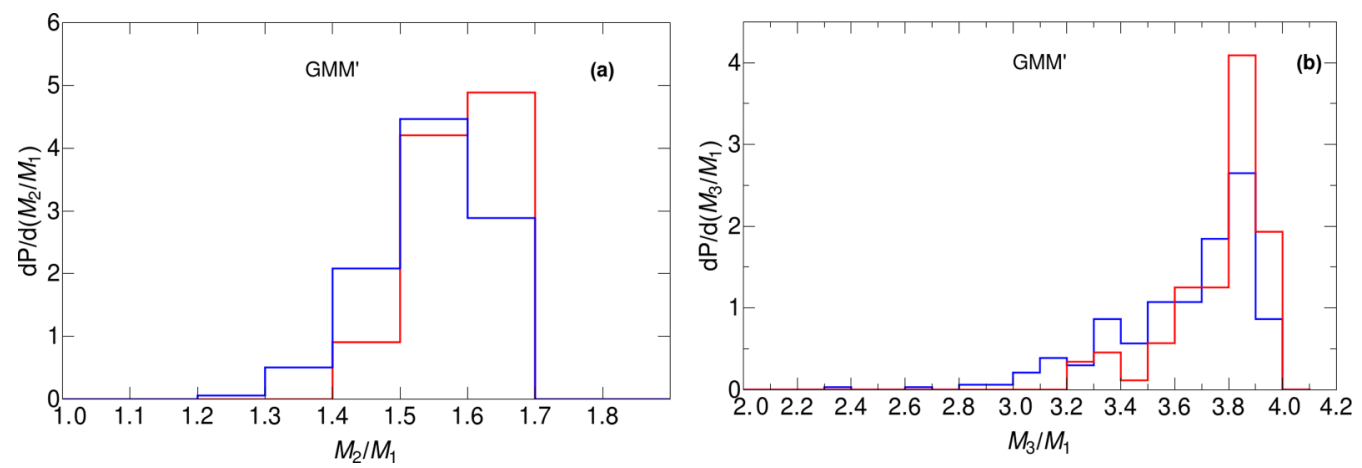

FIG. 6. Distributions in (a) $M_{2} / M_{1}$ and (b) $M_{3} / M_{1}$. Here, $n=1$ (blue) and $n=2$ (red) are from statistical scans over the nGMM model with $m_{3 / 2}=20 \mathrm{TeV}$. 


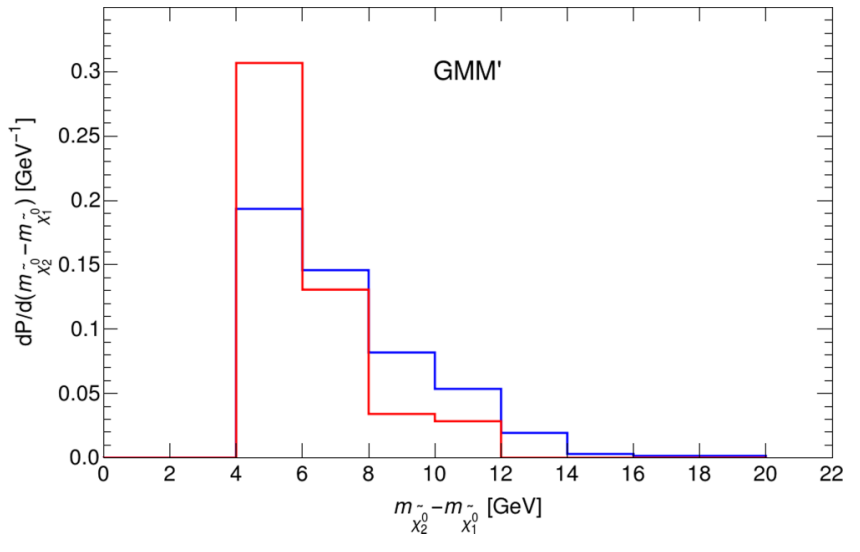

FIG. 7. Probability distribution for light neutral higgsino mass difference $m_{\widetilde{\chi}_{2}^{0}}-m_{\widetilde{\chi}_{1}^{0}}$ from $n=1$ (blue) and $n=2$ (red) statistical scans over the nGMM model with $m_{3 / 2}=20 \mathrm{TeV}$.

top-squark soft terms owing to the large trilinear terms $A_{0}^{\mathrm{MM}} \equiv$ $-a_{3} \alpha\left(m_{3 / 2} / 16 \pi^{2}\right)$. This region is nearly flat with increasing $m_{0}$ mainly because the larger we make the GUT scale topsquark squared mass soft terms, the larger is the canceling correction from RG running. For larger $m_{1 / 2}$ values, then we obtain viable EW vacua since large values of $M_{3}$ help to enhance top-squark squared mass running to large positive values (see, e.g., Eq. (9.16h) of Ref. [64]). The dots show the expected statistical result of scanning the landscape, and the larger density of dots on the plot corresponds to greater stringy naturalness. We also show the magenta contour of $m_{\tilde{g}}=2.25 \mathrm{TeV}$, below which is excluded by LHC gluino pair searches. We also show contours of $m_{h}=123$ and $125 \mathrm{GeV}$. The green points are consistent with LHC sparticle search limits and Higgs mass measurement. From the plot, we see
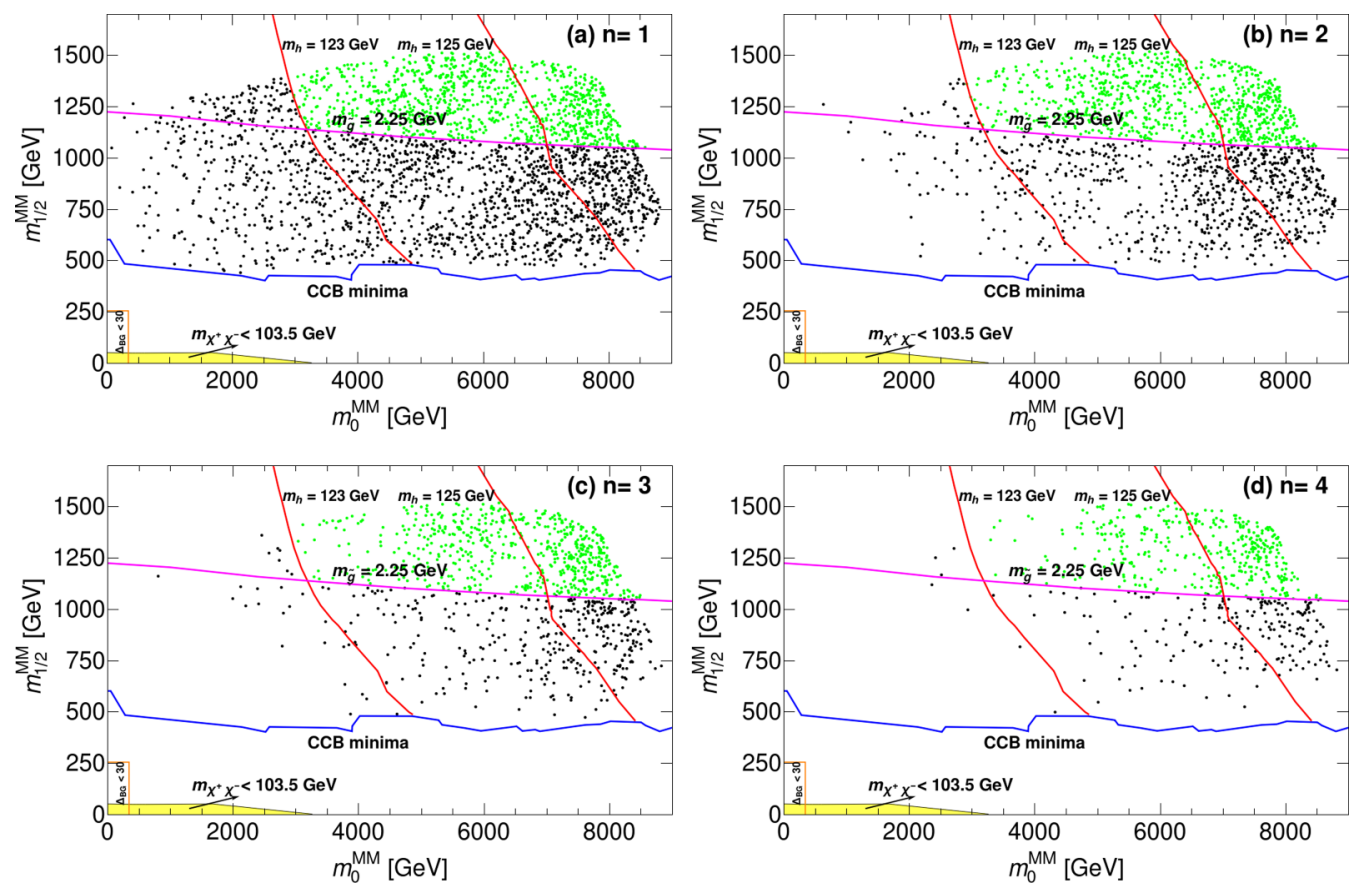

FIG. 8. For $m_{3 / 2}=20 \mathrm{TeV}$, we plot the GMM parameter space in the $m_{0}^{\mathrm{MM}}$ vs $m_{1 / 2}^{\mathrm{MM}}$ parameter space for $a_{3}=1.6 \sqrt{c_{m}}$ with $c_{m 3}=c_{m}$ and $\tan \beta=10$ with $m_{A}=2 \mathrm{TeV}$. We plot for a landscape draw of (a) $n=1$, (b) 2 , (c) 3 , and (d) 4 with $m_{Z}^{\mathrm{PU}}<4 m_{Z}^{\mathrm{OU}}$. 
TABLE I. Input parameters and masses in $\mathrm{GeV}$ units for a natural mirage mediation SUSY benchmark point as compared to a similar point from the NUHM2 model with $m_{t}=173.2 \mathrm{GeV}$. The input parameters for the natural mirage mediation model such as $\alpha$ and $c_{m}$ have been calculated from $m_{0}^{\mathrm{MM}}$ and $m_{1 / 2}^{\mathrm{MM}}$ which are taken equal to $m_{0}$ and $m_{1 / 2}$ respectively as in NUHM2 model. The $c_{m}$ and $c_{m 3}$ have been taken equal to each other so that masses of first/second and third generation sfermions are equal at the GUT scale so as to match the NUHM2 model.

\begin{tabular}{|c|c|c|}
\hline parameter & NUHM2 & $\mathrm{GMM}^{\prime}$ \\
\hline$m_{0}$ & 5000 & -- \\
\hline$m_{1 / 2}$ & 1250 & -- \\
\hline$A_{0}$ & -8000 & -- \\
\hline $\tan \beta$ & 10 & 10 \\
\hline$m_{3 / 2}$ & -- & 20000 \\
\hline$\alpha$ & -- & 9.9 \\
\hline$c_{m}$ & -- & 16 \\
\hline$c_{m 3}$ & -- & 16 \\
\hline$a_{3}$ & -- & 6.4 \\
\hline$\mu$ & 200 & 200 \\
\hline$m_{A}$ & 2000 & 2000 \\
\hline$m_{\tilde{g}}$ & 2931.4 & 2556.5 \\
\hline$m_{\tilde{u}_{L}}^{\circ}$ & 5479.6 & 5305.3 \\
\hline$m_{\tilde{u}_{R}}$ & 5598.3 & 5432.8 \\
\hline$m_{\tilde{e}_{R}}$ & 4822.6 & 4827.9 \\
\hline$m_{\tilde{t}_{1}}$ & 1750.2 & 1646.2 \\
\hline$m_{\tilde{t}_{2}}$ & 3953.6 & 3803.6 \\
\hline$m_{\tilde{b}_{1}}$ & 3987.4 & 3836.7 \\
\hline$m_{\tilde{b}_{2}}$ & 5322.1 & 5169.5 \\
\hline$m_{\tilde{\tau}_{1}}$ & 4745.2 & 4752.2 \\
\hline$m_{\tilde{\tau}_{2}}$ & 5116.3 & 5094.0 \\
\hline$m_{\tilde{v}_{\tau}}$ & 5122.8 & 5101.0 \\
\hline$m_{\widetilde{\chi}_{2}^{ \pm}}$ & -1061.2 & -1116.9 \\
\hline$m_{\tilde{\chi}_{1}^{ \pm}}$ & -210.0 & -210.1 \\
\hline$m_{\widetilde{\chi}_{4}^{0}}$ & -1074.7 & -1129.9 \\
\hline$m_{\widetilde{\chi}_{3}^{0}}$ & -562.3 & -748.5 \\
\hline$m_{\widetilde{\chi}_{2}^{0}}$ & 208.2 & 207.8 \\
\hline$m_{\widetilde{\chi}_{1}^{0}}$ & -198.3 & -199.7 \\
\hline$m_{h}$ & 124.8 & 124.2 \\
\hline$\Omega_{\widetilde{\chi}_{1}^{0}}^{s t d} h^{2}$ & 0.011 & 0.010 \\
\hline$B F(b \rightarrow s \gamma) \times 10^{4}$ & 3.1 & 3.1 \\
\hline$B F\left(B_{s} \rightarrow \mu^{+} \mu^{-}\right) \times 10^{9}$ & 3.8 & 3.8 \\
\hline$\sigma^{S I}\left(\widetilde{\chi}_{1}^{0}, p\right)(\mathrm{pb})$ & $0.16 \times 10^{-8}$ & $0.11 \times 10^{-8}$ \\
\hline$\sigma^{S D}\left(\widetilde{\chi}_{1}^{0} p\right)(\mathrm{pb})$ & $0.33 \times 10^{-4}$ & $0.21 \times 10^{-4}$ \\
\hline$\left.\langle\sigma v\rangle\right|_{v \rightarrow 0}\left(\mathrm{~cm}^{3} / \mathrm{sec}\right)$ & $0.2 \times 10^{-24}$ & $0.2 \times 10^{-24}$ \\
\hline$\Delta_{\mathrm{EW}}$ & 24.4 & 18.2 \\
\hline
\end{tabular}

choosing $m_{3 / 2}=20 \mathrm{TeV}$. From Table I, we see that the scalar mass spectrum is heavy and rather similar for the two cases. For the gaugino spectrum, we see that while $m_{\widetilde{\chi}_{2}^{ \pm}} \sim m_{\widetilde{\chi}_{4}^{0}} \sim M_{2} \sim 1100 \mathrm{GeV}$ for both models, the gluino mass $m_{\tilde{g}} \sim 2556 \mathrm{GeV}$ for $\mathrm{GMM}^{\prime}$ which is rather less than the value $m_{\tilde{g}} \sim 2931 \mathrm{GeV}$ for NUHM2. Also, we see that $m_{\widetilde{\chi}_{3}^{0}} \sim M_{1} \sim 748 \mathrm{GeV}$ for $\mathrm{GMM}^{\prime}$ while $m_{\widetilde{\chi}_{3}^{0}} \sim 562 \mathrm{GeV}$ for NUHM2. Thus, the gaugino masses are compressed in GMM' compared to the gauginos from NUHM2 with a universal value of $m_{1 / 2}$ at $m_{\mathrm{GUT}}$. Both models have a cluster of higgsinos around $\mu \sim 200 \mathrm{GeV}$ so these models may be difficult to distinguish at LHC upgrades. It may require an $e^{+} e^{-}$collider operating with $\sqrt{s}>2 m$ (higgsino) to measure the gaugino masses indirectly via their contribution to higgsino mass splitting. Such a collider could then distinguish mirage unification of gauginos compared to GUT scale unified gaugino masses [59].

\section{CONCLUSIONS}

From rather general considerations of the string landscape, it is to be expected that there is a statistical power-law preference $m_{\text {soft }}^{n}$ for soft SUSY breaking terms as large as possible, subject to the anthropic condition that electroweak symmetry is properly broken and that the pocket universe value of the weak scale does not exceed a factor 2-5 (here we use 4) from its measured value in our universe. Such a scenario is apt to lift the gravitino mass $m_{3 / 2}$ into the tens of $\mathrm{TeV}$ range such that AMSB SSB terms are comparable to the weak scale. In such a case, then one expects modulus-mediated and anomaly-mediated soft terms to be comparable and in such a setting the appropriate $N=1$ SUGRA framework is that of generalized mirage mediation.

Within the GMM model and including a natural solution to the SUSY $\mu$ problem, we have made statistical predictions for model parameters and sparticle and Higgs boson mass values for the cases of $n=1$ and 2 with $m_{3 / 2}=20 \mathrm{TeV}$. For $n=1$ with $m_{3 / 2}=20 \mathrm{TeV}$, we find the mirage mediation scale $\mu_{\mathrm{mir}} \sim 10^{10}-2 \times 10^{14} \mathrm{GeV}$, while for $n=$ 2 then $\mu_{\text {mir }} \sim 8 \times 10^{12}-3 \times 10^{14} \mathrm{GeV}$. These predictions can be somewhat falsified by measuring the gaugino masses at LHC or a high energy $e^{+} e^{-}$collider and extrapolating their masses via renormalization group running to find their intersection point $\mu_{\text {mir }}$, which then determines the mixing parameter $\alpha$. In this happy event, then one could also $d i$ rectly extract the gravitino mass $m_{3 / 2}$. The mirage-mediation scenario would be rather implausible if no mirage mediation scale was found (the three gaugino masses did not unify at a point) or if $\mu_{\text {mir }}$ was found to lie outside these ranges.

Regarding Higgs and sparticle mass predictions, the light Higgs boson mass is found to peak rather sharply around $m_{h} \simeq 125 \mathrm{GeV}$. This is understood in part because the trilinear SSB term is pulled to large-but not too large-values such that there is large mixing in the stop sector leading to large radiative corrections to $m_{h}$. The Higgs mass cannot get too large lest SUSY radiative corrections to the weak scale drive the value of the pocket-universe weak scale $m_{Z}^{\mathrm{PU}}$ beyond the Agrawal et al. [8] anthropic window of allowed values.

Meanwhile, the gluino is pulled up to $m_{\tilde{g}} \sim 3.5 \pm 1.5 \mathrm{TeV}$ and the light top squark is pulled to $m_{\tilde{t}_{1}} \sim 1.5 \pm 0.5 \mathrm{TeV}$. With such large values of $m_{\tilde{g}}$ and $m_{\tilde{t}_{1}}$, an energy upgrade of LHC may be needed to realize SUSY discovery via gluino and/or top-squark pair production. The pseudoscalar Higgs boson $m_{A} \sim 3.5 \pm 1.5 \mathrm{TeV}$ so it seems typically beyond the projected reach of LHC luminosity upgrades. The most likely avenue for SUSY discovery at LHC would be via direct Higgsino pair production $p p \rightarrow \tilde{\chi}_{1}^{0} \tilde{\chi}_{2}^{0} \rightarrow \ell^{+} \ell^{-}+E_{/ T}$ where 
the presence of an initial state jet radiation may help to trigger on the expected soft dilepton signature [62]. The soft dilepton invariant mass is expected to be bounded by $m_{\widetilde{\chi}_{2}^{0}}-m_{\widetilde{\chi}_{1}^{0}} \sim$ 5-10 GeV. In fact, such a soft opposite-sign dilepton excess seems to be building in Atlas data. Precision measurement of higgsino pair production also presents excellent motivation for construction of an $e^{+} e^{-}$collider with $\sqrt{s}>2 m$ (higgsino $) \simeq$ $2 \mu \simeq 400-600 \mathrm{GeV}$ [59].

\section{ACKNOWLEDGMENTS}

H.B. thanks the University of Colorado Department of Physics and Astrophysics for hospitality while this work was completed. D.S. thanks the Fermilab Theory Group for hospitality when part of this work was done. This material is based upon work supported by the U.S. Department of Energy, Office of Science, Office of High Energy Physics under Award No. DE-SC-0009956.
[1] S. Weinberg, Phys. Rev. Lett. 59, 2607 (1987).

[2] S. Weinberg, Rev. Mod. Phys. 61, 1 (1989).

[3] A. Linde, Rep. Prog. Phys. 80, 022001 (2017).

[4] S. Weinberg, in Universe or Multiverse? edited by B. Carr (Cambridge University Press, Cambridge, 2007), pp. 29-42.

[5] R. Bousso and J. Polchinski, J. High Energy Phys. 06 (2000) 006.

[6] L. Susskind, in Universe or Multiverse? edited by B. Carr (Cambridge University Press, Cambridge, 2007), pp. 247-266.

[7] S. Ashok and M. R. Douglas, J. High Energy Phys. 01 (2004) 060.

[8] V. Agrawal, S. M. Barr, J. F. Donoghue, and D. Seckel, Phys. Rev. Lett. 80, 1822 (1998); Phys. Rev. D 57, 5480 (1998).

[9] J. F. Donoghue, in Universe or Multiverse? edited by B. Carr (Cambridge University Press, Cambridge, 2007), pp. 231-246.

[10] M. R. Douglas, arXiv:hep-th/0405279.

[11] H. Baer, V. Barger, M. Savoy, and H. Serce, Phys. Lett. B 758, 113 (2016).

[12] N. Arkani-Hamed, S. Dimopoulos, and S. Kachru, arXiv:hepth/0501082.

[13] H. Baer, V. Barger, H. Serce, and K. Sinha, J. High Energy Phys. 03 (2018) 002.

[14] D. Matalliotakis and H. P. Nilles, Nucl. Phys. B 435, 115 (1995); M. Olechowski and S. Pokorski, Phys. Lett. B 344, 201 (1995); P. Nath and R. L. Arnowitt, Phys. Rev. D 56, 2820 (1997); J. Ellis, K. Olive, and Y. Santoso, Phys. Lett. B 539, 107 (2002); J. Ellis, T. Falk, K. Olive, and Y. Santoso, Nucl. Phys. B 652, 259 (2003); H. Baer, A. Mustafayev, S. Profumo, A. Belyaev, and X. Tata, J. High Energy Phys. 07 (2005) 065.

[15] H. Baer, V. Barger, S. Salam, H. Serce, and K. Sinha, J. High Energy Phys. 04 (2019) 043.

[16] H. Baer, V. Barger, D. Sengupta, H. Serce, K. Sinha, and R. W. Deal, Eur. Phys. J. C 79, 897 (2019).

[17] H. Baer, V. Barger, and D. Sengupta, Phys. Rev. Res. 1, 033179 (2019).

[18] K. Choi, A. Falkowski, H. P. Nilles, and M. Olechowski, Nucl. Phys. B 718, 113 (2005).

[19] H. Baer, V. Barger, H. Serce, and X. Tata, Phys. Rev. D 94, 115017 (2016).

[20] L. Randall and R. Sundrum, Nucl. Phys. B 557 (1999) 79; G. F. Giudice, M. A. Luty, H. Murayama, and R. Rattazzi, J. High Energy Phys. 12 (1998) 027; J. A. Bagger, T. Moroi, and E. Poppitz, ibid. 04 (2000) 009.

[21] K. Fujii, C. Grojean, M. E. Peskin, T. Barklow, Y. Gao, S. Kanemura, H. Kim, J. List, M. Nojiri, M. Perelstein et al., arXiv:1702.05333.
[22] H. Baer, E. K. Park, X. Tata, and T. T. Wang, Phys. Lett. B 641, 447 (2006).

[23] S. Kachru, R. Kallosh, A. D. Linde, and S. P. Trivedi, Phys. Rev. D 68, 046005 (2003).

[24] K. Choi, A. Falkowski, H. P. Nilles, M. Olechowski, and S. Pokorski, J. High Energy Phys. 11 (2004) 076.

[25] O. Loaiza-Brito, J. Martin, H. P. Nilles, and M. Ratz, in Particles, Strings, and Cosmology: 11th International Symposium on Particles, Strings, and Cosmology; PASCOS 2005, edited by K. Choi, J. E. Kim, and D. Son, AIP Conf. Proc. No. 805 (AIP, New York, 2005).

[26] K. Choi, K. S. Jeong, and K.-i. Okumura, J. High Energy Phys. 09 (2005) 039.

[27] A. Falkowski, O. Lebedev, and Y. Mambrini, J. High Energy Phys. 11 (2005) 034.

[28] K. Choi, K. S. Jeong, T. Kobayashi, and K.-i. Okumura, Phys. Rev. D 75, 095012 (2007).

[29] M. Endo, M. Yamaguchi, and K. Yoshioka, Phys. Rev. D 72, 015004 (2005).

[30] R. Kitano and Y. Nomura, Phys. Lett. B 631, 58 (2005).

[31] H. Baer, E. K. Park, X. Tata, and T. T. Wang, J. High Energy Phys. 08 (2006) 041; 06 (2007) 033.

[32] H. Baer, V. Barger, D. Mickelson, and M. Padeffke-Kirkland, Phys. Rev. D 89, 115019 (2014).

[33] H. Baer, V. Barger, P. Huang, A. Mustafayev, and X. Tata, Phys. Rev. Lett. 109, 161802 (2012).

[34] H. Baer, V. Barger, P. Huang, D. Mickelson, A. Mustafayev, and X. Tata, Phys. Rev. D 87, 115028 (2013).

[35] K. Choi and H. P. Nilles, J. High Energy Phys. 04 (2007) 006.

[36] V. Lowen and H. P. Nilles, Phys. Rev. D 77, 106007 (2008).

[37] J. P. Conlon and F. Quevedo, J. High Energy Phys. 06 (2006) 029.

[38] K. Choi, H. P. Nilles, C. S. Shin, and M. Trapletti, J. High Energy Phys. 02 (2011) 047.

[39] W. Buchmuller, K. Hamaguchi, O. Lebedev, and M. Ratz, Phys. Rev. Lett. 96, 121602 (2006).

[40] W. Buchmuller, K. Hamaguchi, O. Lebedev, and M. Ratz, Nucl. Phys. B 785, 149 (2007).

[41] O. Lebedev, H. P. Nilles, S. Raby, S. Ramos-Sanchez, M. Ratz, P. K. S. Vaudrevange, and A. Wingerter, Phys. Lett. B 645, 88 (2007).

[42] O. Lebedev, H. P. Nilles, S. Raby, S. Ramos-Sanchez, M. Ratz, P. K. S. Vaudrevange, and A. Wingerter, Phys. Rev. Lett. 98, 181602 (2007). 
[43] W. Buchmuller, K. Hamaguchi, O. Lebedev, and M. Ratz, arXiv:hep-ph/0512326.

[44] H. P. Nilles and P. K. S. Vaudrevange, Mod. Phys. Lett. A 30, 1530008 (2015).

[45] H. Baer, V. Barger, M. Savoy, H. Serce, and X. Tata, J. High Energy Phys. 06 (2017) 101.

[46] B. S. Acharya, K. Bobkov, G. Kane, P. Kumar, and D. Vaman, Phys. Rev. Lett. 97, 191601 (2006); B. S. Acharya, K. Bobkov, G. L. Kane, P. Kumar, and J. Shao, Phys. Rev. D 76, 126010 (2007); B. S. Acharya, K. Bobkov, G. L. Kane, J. Shao, and P. Kumar, ibid. 78, 065038 (2008); B. S. Acharya and K. Bobkov, J. High Energy Phys. 09 (2010) 001.

[47] H. Baer, V. Barger, and S. Salam, Phys. Rev. Res. 1, 023001 (2019).

[48] F. E. Paige, S. D. Protopopescu, H. Baer, and X. Tata, arXiv:hep-ph/0312045.

[49] N. Arkani-Hamed and S. Dimopoulos, J. High Energy Phys. 06 (2005) 073.

[50] H. Baer, V. Barger, and D. Sengupta, Phys. Lett. B 790, 58 (2019).

[51] K. J. Bae, H. Baer, V. Barger, and D. Sengupta, Phys. Rev. D 99, 115027 (2019).

[52] S. P. Martin and M. T. Vaughn, Phys. Rev. D 50, 2282(E) (1994); 78, 039903 (2008).

[53] M. Carena and H. E. Haber, Prog. Part. Nucl. Phys. 50, 63 (2003); P. Draper and H. Rzehak, Phys. Rep. 619, 1 (2016).

[54] H. Baer, V. Barger, and A. Mustafayev, Phys. Rev. D 85, 075010 (2012).

[55] H. Baer, V. Barger, J. S. Gainer, P. Huang, M. Savoy, D. Sengupta, and X. Tata, Eur. Phys. J. C 77, 499 (2017).
[56] H. Baer, V. Barger, J. S. Gainer, D. Sengupta, H. Serce, and X. Tata, Phys. Rev. D 98, 075010 (2018).

[57] X. Cid Vidal, M. D'Onofrio, P. J. Fox, R. Torre, K. A. Ulmer, A. Aboubrahim, A. Albert, J. Alimena, B. C. Allanach, C. Alpigiani et al. [Working Group 3], arXiv:1812.07831, CERN Yellow Rep. Monogr. 7, 585 (2019).

[58] K. J. Bae, H. Baer, N. Nagata, and H. Serce, Phys. Rev. D 92 , 035006 (2015).

[59] H. Baer, V. Barger, D. Mickelson, A. Mustafayev, and X. Tata, J. High Energy Phys. 06 (2014) 172; S.-L. Lehtinen, H. Baer, M. Berggren, K. Fujii, J. List, T. Tanabe, and J. Yan, PoS EPS-HEP2017, 306 (2007); K. Fujii, C. Grojean, M. E. Peskin, T. Barklow, Y. Gao, S. Kanemura, H. Kim, J. List, M. Nojiri, M. Perelstein et al., arXiv:1702.05333; H. Baer, M. Berggren, K. Fujii, J. List, S. L. Lehtinen, T. Tanabe, and J. Yan, arXiv:1912.06643.

[60] H. Baer, V. Barger, P. Huang, D. Mickelson, A. Mustafayev, W. Sreethawong, and X. Tata, Phys. Rev. Lett. 110, 151801 (2013).

[61] H. Baer, V. Barger, J. S. Gainer, M. Savoy, D. Sengupta, and X. Tata, Phys. Rev. D 97, 035012 (2018).

[62] H. Baer, V. Barger, and P. Huang, J. High Energy Phys. 11 (2011) 031; Z. Han, G. D. Kribs, A. Martin, and A. Menon, Phys. Rev. D 89, 075007 (2014); H. Baer, A. Mustafayev, and X. Tata, ibid. 90, 115007 (2014); C. Han, D. Kim, S. Munir, and M. Park, J. High Energy Phys. 04 (2015) 132; H. Baer, V. Barger, M. Savoy, and X. Tata, Phys. Rev. D 94, 035025 (2016).

[63] The ATLAS collaboration (ATLAS Collaboration), ATLASCONF-2019-014.

[64] H. Baer and X. Tata, Weak Scale Supersymmetry: From Superfields to Scattering Events (Cambridge University Press, Cambridge, UK, 2006), p. 537. 